\title{
Das ethische Dilemma der «richtigen» Geschlechtszuteilung
}

Redaktion Ethik

Korrespondenz:

Schweizerische Ärztezeitung

Redaktion Ethik

Farnsburgerstrasse 8

$\mathrm{CH}-4132$ Muttenz

redaktion.saez@emh.ch

\section{Vorbemerkung}

Die Schweizerische Ärztezeitung setzt ihre Serie ethischer Falldiskussionen fort, dieses Mal zum heiklen Thema der Geschlechtszuteilung bei einem adrenogenitalen Syndrom. Diese Problematik führt an die Grenzen des Begriffs von Gesundheit und Krankheit, von Normalität und Abweichung, von Individuum und Kultur. Sie übersteigt damit den Fokus der klassischen Medizinethik, die vor allem aus der Arzt-Patienten-Beziehung heraus denkt. Sie betrifft ganz wesentlich auch die gesellschaftlich geprägten Erwartungen an eine geschlechtsbezogene Körperlichkeit. Es geht nicht nur um Funktionsfähigkeit und Wohlbefinden, sondern auch um Geschlechterrollen und Körpernormen, die reich mit kulturellen Bedeutungen gefüllt sind. Für die Betroffenen und ihre Familien sind dies verständlicherweise sehr sensible Themen.

Die Schwierigkeit einer ethischen Diskussion besteht darin, beide Ebenen (Gesellschaft und therapeutische Beziehung) in ein gutes Verhältnis zueinander zu bringen. Sowohl Geschlechterrollen als auch Körpernormen schlagen sich für die einzelnen in sehr starken, z.T. verinnerlichten sozialen Erwartungen nieder. Menschen, deren Körper die Norm durchbricht, verlangen nach der Medizin, um den Körper zu normalisieren. Gleichzeitig hat, wie die Berichte zeigen, die Problematik aber auch einen Aspekt von Gesundheit und Krankheit, der nicht nur von den gesellschaftlichen Normen abhängt.

Auf der einen Seite ist der Patient/die Patientin mit ihren Angehörigen, die bezüglich ihrer geschlechtlichen Identität gerade durch eine Moralisierung der Diskussion verletzbar werden. Auf der anderen Seite ist die ethisch argumentierende Gesellschaftstheorie, die abstrakt bleibt. Muss sich nun aber die abstrakte Idee durch die Konkretheit des Einzelfalles, durch die Menschlichkeit der Personen herausfordern lassen? Der Philosoph Emmanuel Levinas pflegte jedenfalls darauf hinzuweisen, dass es in kasuistischen Diskussionen darum geht, die «grossmütigen allgemeinen Ideen» durch einen Kontakt mit der konkreten Wirklichkeit «vor Entfremdung zu bewahren». Dies soll hier geschehen. Die beiden nachfolgenden Kommentare von Kathrin
Zehnder und Nikola Biller-Andorno greifen die Ideen und die Wirklichkeit der Situation in verschiedener Weise auf und lassen die Diskussion offen.

Der Fall selbst wurde der Redaktion von einem Kollegen mitgeteilt, dessen Name zum Schutz der Anonymität des Patienten und seiner direkt betroffenen Angehörigen ungenannt bleibt. Persönliche Details in der Fallbeschreibung wurden abgeändert.

\section{Problemschilderung: Spätdiagnose eines adrenogenitalen Syndroms und Entscheidungsdilemmata}

\section{Vorgeschichte}

Ein damals 7jähriges Mädchen wurde in die Endokrinologiesprechstunde zugewiesen wegen «zwittrigem Genitale». Es stellte sich heraus, dass das Mädchen an einem bekannten adrenogenitalen Syndrom (AGS) litt, weshalb sie bereits nach der Geburt in ihrem Heimatland bei Vermännlichung des äusseren Genitales operiert worden war. Seit dem Neugeborenenalter hatte das Kind ein Cortisonpräparat und ein Salzhormon als Dauertherapie erhalten. Die Familie des Kindes war etwa 2 Jahre vor der jetzigen $\mathrm{Zu}$ weisung als Flüchtlinge in die Schweiz gekommen, wo offenbar nach Beendigung der Packung die Medikamente nicht mehr weiter gegeben wurden. Das Mädchen war grossgewachsen und laut Ansicht der Eltern bei bester Gesundheit. Doch wegen des fehlenden Cortisonersatzes war es zu einer erneuten Virilisierung des äusseren Genitales gekommen. Zudem zeigte das 7jährige Mädchen bereits eine beschleunigte körperliche Reifung (Knochenalter 11 Jahre) und stand klinisch (Brustentwicklung) und labormässig zu Beginn der Pubertätsentwicklung.

\section{Pathologie}

Beim AGS handelt es sich um einen angeborenen Enzymdefekt in der Synthese der Nebennierenrindenhormone, unter denen Cortisol und Aldosteron lebenswichtig sind. In der Nebenniere werden zudem bei Männern und Frauen auch männliche Hormone gebildet. Diese Androgenproduktion wird beim AGS 
wegen stimulierendem hypophysärem Feedback (ACTH) bei Cortisolmangelsituation übermässig stimuliert, so dass es bereits in utero zu einer mehr oder weniger starken Virilisierung weiblicher Feten kommt. Unbehandelt ist beim Neugeborenen, aber auch im späteren Leben eine lebensgefährliche Entgleisung des Salzhaushaltes zu befürchten. Im Verlauf zeigen die Kinder bei ungenügender Therapie eine avancierte körperliche Reifung und späteren Kleinwuchs als Erwachsene. Durch adäquaten und lebenslänglichen Cortisolersatz kann der Teufelskreis der Androgenisierung mit seinen Folgeerscheinungen unterbrochen werden.

Das AGS wird rezessiv vererbt, wobei es verschiedene Mutationen gibt. Meist sind beide Eltern heterozygote Träger einer Mutation, wodurch jedes Kind mit 25\%-Risiko als homozygoter (oder doppelt heterozygoter) Patient von der Erkrankung betroffen sein kann. In Mitteleuropa ist jeder vierzigste unerkannter Träger einer solchen Mutation, im Balkan und Mittelmeerraum sogar jeder fünfundzwanzigste. In der Schweiz besteht seit 1993 für alle Neugeborenen ein Screening auf 17-OH-Progesteron, so dass Betroffene erkannt und behandelt werden können, bevor eine Entgleisung auftritt.

\section{Der Patient}

Bei einer Verlaufsuntersuchung sollte bei der Patientin und den Eltern eine molekulargenetische Untersuchung durchgeführt werden, da in der Familie weiterer Kinderwunsch bestand. Bei dieser Gelegenheit kam zufällig auch der 5jährige Bruder erstmals mit. Der Knabe war im ersten Kindergartenjahr und schien für sein Alter recht gross zu sein, weshalb er im Einverständnis der Eltern gemessen und kurz untersucht wurde. Der muskulöse Knabe zeigte ein bereits nicht mehr kindliches männliches Genitale mit deutlicher (rasierter) Pubesbehaarung, einem kräftigen Penis ohne Vorhaut und leichter Hypospadie sowie einem gut ausgebildeten, aber leeren Scrotum. Im Ultraschall des inneren Genitales fanden sich ein Uterus und Ovarien beidseits, eine Vagina war nicht darstellbar. Das Handröntgenbild entsprach der Reifung eines 11jährigen Mädchens bzw. 13jährigen Knaben. Die Blutuntersuchungen mit massiv erhöhten Androgenen und Karyotyp 46 XX bestätigten den Verdacht eines bisher nicht erkannten AGS mit vollständiger Virilisierung des äusseren Genitales (Prader V) bei einem «eigentlichen Mädchen» (46 XX) mit intakten inneren weiblichen Strukturen. Zusätzlich hatte die jahrelange Androgenproduktion zu einer starken Avancierung der Reifung mit Auslösung der spontanen Pubertätsent- wicklung geführt. Durch die reifungsbedingte Aktivierung der Hypophyse hatte die Östrogenproduktion der Ovarien begonnen, so dass «der Knabe» nach wenigen Monaten unter Therapie auch mit der weiblichen Brustentwicklung begann. Beide Kinder wurden wegen der vorzeitigen Reifung (Pubertas praecox vera) zusätzlich zum Cortisonersatz mit einem hypophysär hemmenden LHRH analog behandelt, das die Ovarialstimulation unterdrücken sollte. Die molekulargenetische Untersuchung ergab, dass beide Kinder von ihren heterozygoten Eltern die gleiche homozygote AGS-Mutation bekommen hatten.

\section{Familiäre/kulturelle Situation}

Die Familie war aus ihrem Heimatland als Flüchtlinge in die Schweiz gekommen, da einige Verwandte schon früher hier Aufnahme gefunden hatten. Der Vater verstand mässig gut Deutsch, die Mutter hingegen kein Wort. Die beiden älteren, AGS-betroffenen Kinder besuchten die Primarschule bzw. den Kindergarten und waren recht gut integriert. Das dritte, inzwischen geborene Kind der Familie war auch ein Mädchen (heterozygote Trägerin, selbst nicht krank). Die Familie war moslemischer Glaubensrichtung.

\section{Ethisches Dilemma}

Ein zuvor als gesund und kräftig geltender Knabe wird «zufällig» als an sich schwer AGS-krankes Mädchen mit vollständiger äusserer Vermännlichung erkannt. Die Kernfrage bestand darin, dem Kind jetzt - aber im Hinblick auf seine künftigen Lebensphasen (Kindheit, Adoleszenz, Erwachsenenalter) - die «richtige Geschlechtszuordnung» zu geben, damit es jetzt und später möglichst wohl und in seiner Geschlechtsrolle sicher und zufrieden aufwachsen kann.

Liesse man das Kind weiter als Knaben aufwachsen, so würden die inneren weiblichen Genitalanlagen mit bereits vorhandener Aktivierung (Brustentwicklung) «stören», so dass eine Entfernung von Uterus und Ovarien in Frage käme. Damit wäre aber ein definitiver Verlust der Fertilität verbunden. Entsprechend würde der Knabe ab Pubertäts-/Erwachsenenalter einen lebenslänglichen Testosteronersatz und evtl. die Einlage von Hodenprothesen benötigen. Die sexuelle Funktion als Erwachsener wäre unsicher, wahrscheinlich aber möglich, da gute Schwellkörperbildung vorhanden ist (ohne Samenerguss). Wegen der avancierten Reifung ist die Wachstumsreserve des Patienten beschränkt, so dass eine Körpergrösse um $160 \mathrm{~cm}$ resultieren dürfte. 
Liesse man das Kind als Mädchen aufwachsen, so würde dies Korrekturoperationen des Genitales mit Reduktion der stark vergrösserten Klitoris und eine Neubildung der Vagina bedeuten. Die Fertilität wäre möglich, doch wäre der Wechsel der Geschlechtsorientierung in diesem Alter sicher sehr heikel. Die männliche Prägung des kindlichen Gehirnes durch die intrauterine Androgenexposition und das bisherige Aufwachsen in der Rolle des Knaben wären nur bedingt korrigierbar und die psychischen Auswirkungen auf das Kind enorm. Die sexuelle Orientierung als Erwachsene wäre evtl. schwierig, wie auch bei frühoperierten virilisierten Mädchen später fehlende, unzufriedene oder gleichgeschlechtliche Beziehungen häufiger sind.

Es besteht also das Dilemma des Wechsels der aktuellen Geschlechtsorientierung versus Fertilitätsverlust. Das bedeutet auch, dass die ethischen Konzepte der Autonomie und des «Nichtschadens» gegeneinander abgewogen werden müssen.

Das kindliche Alter des Patienten bedingt, dass der aktuell 6jährige gar nicht recht mitentscheiden kann, dass also Eltern und beteiligte Ärzte für sein späteres Wohl entscheiden müssen (Konzept der Beneficience). Die Einschätzung der jetzigen und späteren Geschlechtsidentität ist in diesem Alter fast nicht möglich.

Zudem geht die Herkunft der Eltern aus dem moslemischen Land mit einer sehr schicksalsergebenen Haltung und einer Passivität im Entscheidungsprozess einher, so dass der Entscheid fast vollständig dem behandelnden Arzt übergeben wird.

\section{Weiterer Verlauf}

In vielen ausführlichen Gesprächen mit den Eltern, mit Hilfe der Übersetzung z.T. durch Familienmitglieder, später auch durch einen externen Übersetzer, ging es darum, dass die Eltern die ganze Tragweite der Situation und des Prozederes ganz verstehen und akzeptieren konnten. Es zeigte sich bald, dass das Kind als einziger Knabe in seiner Familie eine besondere Stellung als männlicher Nachkomme hatte, die ihm auch nach Aufklärung der Eltern über die chromosomale und anatomische Situation erhalten blieb. Die mögliche Umwandlung des Kindes zu einem Mädchen und damit die Erhaltung der Fortpflanzungsfähigkeit wurde von den Eltern eindeutig weniger stark gewichtet. Hier kommen die ethnische Herkunft und der muslimische Glaube der Familie zum Tragen, so dass der Knabe wegen der wichtigen männlichen Rolle in seiner Gesellschaft auch als «unvollständiger Mann» seine «Wertigkeit» behalten wird. Die genaue Aufklä- rung der Eltern führte auch zu einer aktiveren Haltung der Eltern im Entscheidungsprozess.

Der Knabe selbst konnte bisher nur bedingt über seine Krankheit orientiert werden. Er weiss, dass er an der gleichen Hormonstörung leidet wie seine Schwester und dass (wie bei ihr bereits geschehen) gelegentlich eine Operation durchgeführt werden muss (Korrektur der Hypospadie, gleichzeitig auch Hysterektomie [?] und Ovariektomie oder Korrektur des äusseren Genitales). Mittels langfristig ausgelegter kinderpsychiatrischer Begleitung soll versucht werden, den Knaben in seiner Rolle zu begleiten und zu bestärken.

Die wiederholte Diskussion des Falles unter Fachkollegen und Kinderchirurgen sowie der Literaturvergleich zeigten auf, dass ähnlich gelagerte Fälle, in denen ein Kind mit später AGSDiagnose als Knabe belassen wurde, oft aus dem muslimischen Glaubensbereich kamen.

Theoretisch könnte die Entscheidung der operativen Korrektur vertagt und der Knabe im Alter von etwa 15 Jahren in den Entscheidungsprozess miteinbezogen werden. Dies bedeutet aber eine Weiterführung der aktuellen Bremsung der Ovarialstimulation mit dem Risiko einer dennoch beginnenden Brustentwicklung. Das heisst, der Vorentscheid, den Knaben in der männlichen Rolle weiter aufwachsen zu lassen, fände dennoch statt und die gesellschaftliche Prägung in der männlichen Rolle ginge weiter.

Die Vorgespräche mit dem Übersetzer aus der gleichen Ethnie brachten zusätzliche interessante Aspekte ein: In der Gesellschaft der Herkunftsregion der Familie spielt die Erbschaftsordnung eine wichtige Rolle. Erbberechtigt sind nur männliche Nachkommen, was erneut die Wertigkeit eines Sohnes bestätigt. Als Folge werde dort in Familien ohne männlichen Nachkommen eine der Töchter mit einer männlichen Rolle betraut, die sie nach aussen zu spielen hat. Das heisst, sie trägt Männerkleider und bewegt sich in Männerkreisen, darf aber nicht heiraten. Interessanterweise wird auch einer solchen «Mannfrau» innerhalb des Clans ein Kind als eigener Nachkomme zugeteilt.

Dieser neue Aspekt der Umwandlung einer Frau zu einem Mann (wenn auch aus ganz anderen Motivationsgründen heraus) stellte eine Parallele zum betroffenen Knaben dar und unterstützt die Entscheidungsvariante, den Knaben in seiner bisherigen Rolle weiter aufwachsen zu lassen. Bei der Gesamtbeurteilung der Situation zum Wohl des Patienten spielen also neben anatomischen und medizinischen Überlegungen auch die kulturell-ethnischen Aspekte eine entscheidende Rolle. 